\title{
Carbapenemase detection by NG-Test CARBA 5-a rapid immunochromatographic assay in carbapenem-resistant Enterobacterales diagnosis
}

\author{
Ying Zhu ${ }^{1,2 \#}$, Peiyao Jia ${ }^{1,2 \#}$, Xue Li ${ }^{1,3 \#}$, Tong Wang ${ }^{1}$, Jingjia Zhang ${ }^{1}$, Ge Zhang ${ }^{1}$, Simeng Duan ${ }^{1}$, Wei Kang ${ }^{1}$, \\ Yingchun $\mathrm{Xu}^{1}$, Qiwen Yang ${ }^{1}$ \\ ${ }^{1}$ Department of Clinical Laboratory, State Key Laboratory of Complex Severe and Rare Diseases, Peking Union Medical College Hospital, Chinese \\ Academy of Medical Sciences and Peking Union Medical College, Beijing, China; ${ }^{2}$ Graduate school, Peking Union Medical College, Chinese \\ Academy of Medical Sciences, Beijing, China; ${ }^{3}$ Department of Clinical Laboratory, Beijing Anzhen Hospital, Capital Medical University, Beijing, \\ China \\ Contributions: (I) Conception and design: Q Yang; (II) Administrative support: Y Xu; (III) Provision of study materials or patients: Q Yang, Y Xu; (IV) \\ Collection and assembly of data: T Wang, J Zhang, G Zhang, S Duan, W Kang; (V) Data analysis and interpretation: Q Yang, Y Zhu, P Jia, X Li; (VI) \\ Manuscript writing: All authors; (VII) Final approval of manuscript: All authors. \\ \#These authors contributed equally to this work. \\ Correspondence to: Qiwen Yang. Department of Clinical Laboratory, State Key Laboratory of Complex Severe and Rare Diseases, Peking Union Medical \\ College Hospital, Chinese Academy of Medical Science and Peking Union Medical College, Beijing, China. Email: yangqiwen81@vip.163.com.
}

Background: The global spread of carbapenem-resistant Enterobacterales (CRE) represents a serious public health concern as these organisms are associated with limited treatment options, high mortality rate and rapid transmissibility. The identification of carbapenemase remains a challenge in microbiological laboratories as no single method is perfect when considering cost, carbapenemase coverage, accuracy, handling complexity and TATs together.

Methods: NG-Test CARBA 5 assay and modified carbapenem inactivation method in conjunction with EDTA carbapenem inactivation method (mCIM/eCIM) were challenged with a collection of 299 molecularly characterized CRE isolates in China in order to evaluate the performance in detecting five major carbapenemases ( $b l a_{\mathrm{KPC}}, b l a_{\mathrm{NDM}}, b l a_{\mathrm{VIM}}, b l a_{\mathrm{IMP}}$, and $b l a_{\mathrm{OXA}-48}$ ) among Enterobacterales.

Results: NG-Test CARBA 5 detected all KPC-, NDM-, VIM- and OXA-48-producing isolates perfectly with a weak false-positive signal for NDM in an IMP-4 producer, which makes the specificity for NDM decreases to $99.6 \%$. The overall specificity/sensitivity were $99.9 \% / 100 \%$ for NG-Test CARBA 5. mCIM/ eCIM achieved high specificity of $100 \% / 100 \%$ and sensitivity of $99.6 \% / 97.4 \%$, with one S. marcescens isolate harboring VIM-2 undetected.

Conclusions: Both NG-Test CARBA 5 and mCIM/eCIM showed excellent results in the tested carbapenemase ( $b l a_{\mathrm{KPC}}, b l a_{\mathrm{NDM}}, b l a_{\mathrm{VIM}}, b l a_{\mathrm{IMP}}$, and $\left.b l a_{\mathrm{OXA}-48}\right)$ detection compared with molecular genotypic test. As every assay has its own limitations, suitable methods should be combined for the establishment of the CRE diagnostic pathways.

Keywords: Carbapenemase-resistant Enterobacterales (CRE); EDTA carbapenem inactivation method (eCIM); modified carbapenem inactivation method (mCIM); NG-Test CARBA 5

Submitted Dec 29, 2020. Accepted for publication Mar 05, 2021.

doi: 10.21037/atm-20-8216

View this article at: http://dx.doi.org/10.21037/atm-20-8216 


\section{Introduction}

The global spread of carbapenem-resistant Enterobacterales (CRE) represents a serious public health concern as these pathogens are associated with limited treatment options, high mortality rate and rapid transmissibility $(1,2)$. Mechanisms underlying carbapenem resistance are related mainly to the production of carbapenemases (carbapenemase-producing Enterobacterales, CP-CRE), but also a consequence of decreased membrane permeability coupled with the overexpression of extended-spectrum $\beta$-lactamases (ESBLs) or AmpC $\beta$-lactamases (non-carbapenemase-producing carbapenem-resistant Enterobacterales, non-CP-CRE) (3-5). Detection of the mechanisms of carbapenem resistance is not routinely performed in many Chinese hospitals. However, the distinction between CP-CRE and non-CPCRE is valuable for infection control as CP-CRE can spread rapidly through horizontal gene transfer among Gram-negative organisms and therefore may require implementation of intensive infection control interventions $(3,5)$. Moreover, novel antimicrobial agents possess unique profiles in their activities against different carbapenemases. Ceftazidime-avibactam is active on organisms harboring serine-carbapenemase, but shows no effects on metallo- $\beta$ lactamase (MBL) producers (6-8). Meropenem-vaborbactam has activity against KPC-producing pathogens, while it remains ineffective to OXA-48-like and MBL-producing Enterobacterales (9). Therefore, the identification of carbapenemases is important for making effective treatment decisions.

A variety of phenotypic and genotypic tests can be chosen for CP-CRE detection (10). Modified carbapenem inactivation method in conjunction with EDTA carbapenem inactivation method ( $\mathrm{mCIM} / \mathrm{eCIM})$, recommended by Clinical and Laboratory Standards Institute (CLSI) guidelines, yields high accuracy in carbapenemase detecting, but has long turn-around times (TATs) (3). Molecular genetic approach is regarded as the reference standard and allows the rapid identification of carbapenemase genes either in cultured bacteria or directly from clinical samples (5). However, its performance can be limited by the presence of new variants or rare enzymes which are not detectable by molecular tests $(2,11)$. Furthermore, the expensive instrument and high cost of its reagent restrict the widely application of molecular tests, including GeneXpert $(12,13)$. A newly developed lateral flow immunoassay, NGTest CARBA 5 (NG-Biotech, France), can detect the five most prevalent carbapenemases of KPC, NDM, IMP,
VIM and OXA-48-like within 15 minutes. High sensitivity (from $97.3 \%$ to $100 \%$ ) and specificity (from $96.1 \%$ to $100 \%$ ) of NG-Test CARBA 5 have been reported from studies conducted mainly in Europe (14-19). With simple experimental procedures and short TATs, NG-Test CARBA 5 is a promising method for the CPE detection.

The aim of this study was to compare the performance of NG-Test CARBA 5 and mCIM/eCIM in detecting five major carbapenemases among Enterobacterales with molecular method.

We present the following article in accordance with the STARD reporting checklist (available at http://dx.doi. org/10.21037/atm-20-8216).

\section{Methods}

\section{Bacterial isolates}

A total of 301 isolates were employed in this study, comprising 299 clinical CRE isolates and two quality control (QC) isolates. The 299 clinical CRE isolates, which were collected from respiratory, sterile fluids, tissues, blood, rectal swab/stool, urine, etc., were referred by 20 hospitals in China, from 2007 to 2018, to Peking Union Medical College Hospital (PUMCH). All the Enterobacterales isolates were identified by MALDI-TOF MS (Bruker Biotyper; Bruker Daltonik, Bremen, Germany). CRE was defined as non-susceptible to imipenem, non-susceptible to meropenem or resistant to ertapenem, by the broth microdilution method as per breakpoints criteria of CLSI M100-S30 (20). Isolates recovered from frozen stock $\left(-80{ }^{\circ} \mathrm{C}\right)$ were subcultured twice on blood agar plate with a meropenem disk placed and incubated at $35 \pm 2{ }^{\circ} \mathrm{C}$ overnight to ensure purity.

\section{Molecular detection of carbapenemase genes}

$b l a_{\mathrm{KPC}}, b l a_{\mathrm{NDM}}, b l a_{\mathrm{VIM}}, b l a_{\mathrm{IMP}}$, and $b l a_{\mathrm{OXA}-48}$ genes were detected by multiplex PCR according to Poirel et al. (21). Non-CP-CRE were further analyzed for the presence of plasmid-mediated AmpC $\beta$-lactamase or ESBL genes $b l a_{\mathrm{TEM}}, b l a_{\mathrm{SHV}}, b l a_{\mathrm{CTX}-\mathrm{M}}, b l a_{\mathrm{CMY}}$ and $b l a_{\mathrm{DHA}}(22,23)$. All positive isolates were sent for subsequent DNA sequencing and the obtained gene sequences were compared with NCBI nucleotide database using blast server (http://blast.ncbi. nlm.nih.gov). Sequences were considered a match if $100 \%$ sequence identity of coding sequence (CDS) and 100\% coverage were present (11). 


\section{NG-Test CARBA 5}

The tests were performed as per manufacturer's instructions. Briefly, a $1 \mu \mathrm{L}$ loopful of bacteria was mixed with five drops of extraction buffer, and $100 \mu \mathrm{L}$ of the mixture was dispensed into the CARBA- 5 cassette. Results were interpreted after 15 min of incubation at room temperature (3).

\section{$m C I M / e C I M$}

The tests were performed and interpreted as per instructions from CLSI M100-S30 (20). Klebsiella pneumoniae ATCC BAA-1706 and ATCC BAA-1705 were used as negative and positive quality controls, respectively.

\section{Statistical analysis}

The investigators performing NG-Test CARBA 5 and $\mathrm{mCIM} / \mathrm{eCIM}$ were blind to the identities of the isolates. Sensitivity and specificity were calculated with a $95 \%$ confidence interval (CI) based on an exact binomial distribution. SPSS, version 15.0 (SPSS Inc., Chicago, USA) was used for data analysis.

\section{Ethical statement}

The study was conducted in accordance with the Declaration of Helsinki (as revised in 2013). The study was approved by The Human Research Ethics Committee of Peking Union Medical College Hospital of S-K1056 and informed consent was waived as the study collected remaining samples from clinical tested samples without extra operation of patients.

\section{Results}

\section{Distribution of species and carbapenemases}

Eight species were identified in 299 CRE isolates, including 175 Klebsiella pneumoniae (58.5\%), 48 Escherichia coli (16.1\%), 46 Enterobacter cloacae (15.4\%), 9 Klebsiella oxytoca (3.0\%), 8 Klebsiella aerogenes (2.7\%), 8 Citrobacter freundii (2.7\%), 4 Serratia marcescens (1.3\%), and 1 Proteus mirabilis $(0.3 \%)$. A total of 253 isolates were positive for carbapenemase genes, among which 172 KPC (68.0\%), $29 \operatorname{NDM}(11.5 \%), 41$ IMP (16.2\%), 3 OXA-48 (1.2\%), 4 VIM (1.6\%), 2 IMP and VIM co-existing (0.8\%), as well as $2 \mathrm{KPC}$ and IMP co-existing $(0.8 \%)$ genes were identified (Table 1).

Table 1 Overview of the 299 clinical CRE isolates tested for the evaluation of NG-Test CARBA 5 and mCIM/eCIM

\begin{tabular}{|c|c|c|c|c|c|c|c|c|c|}
\hline Carbapenemase & $\begin{array}{c}\text { Klebsiella } \\
\text { pneumoniae }\end{array}$ & $\begin{array}{c}\text { Escherichia } \\
\text { coli }\end{array}$ & $\begin{array}{l}\text { Klebsiella } \\
\text { aerogenes }\end{array}$ & $\begin{array}{c}\text { Enterobacter } \\
\text { cloacae }\end{array}$ & $\begin{array}{c}\text { Klebsiella } \\
\text { oxytoca }\end{array}$ & $\begin{array}{c}\text { Citrobacter } \\
\text { freundii }\end{array}$ & $\begin{array}{c}\text { Proteous } \\
\text { mirabilis }\end{array}$ & $\begin{array}{c}\text { Serratia } \\
\text { marcescens }\end{array}$ & Total \\
\hline \multicolumn{10}{|l|}{ CP-CRE $(n=253)$} \\
\hline \multicolumn{10}{|l|}{ KPC $(n=172)$} \\
\hline KPC-2 & 132 & 24 & 4 & 5 & 1 & & 1 & 3 & 170 \\
\hline KPC-3 & 1 & & & & & & & & 1 \\
\hline KPC-4 & 1 & & & & & & & & 1 \\
\hline \multicolumn{10}{|l|}{ NDM (n=29) } \\
\hline NDM-1 & 6 & 4 & & 15 & 1 & 1 & & & 27 \\
\hline NDM-4 & & 1 & & & & & & & 1 \\
\hline NDM-5 & & 1 & & & & & & & 1 \\
\hline \multicolumn{10}{|l|}{ IMP $(n=41)$} \\
\hline IMP-1 & 1 & 2 & & 2 & 1 & 4 & & & 10 \\
\hline IMP-4 & 7 & & 1 & 10 & 4 & 2 & & & 24 \\
\hline IMP-8 & 4 & & & 1 & & & & & 5 \\
\hline IMP-26 & 1 & & & 1 & & & & & 2 \\
\hline
\end{tabular}

Table 1 (continued) 
Table 1 (continued)

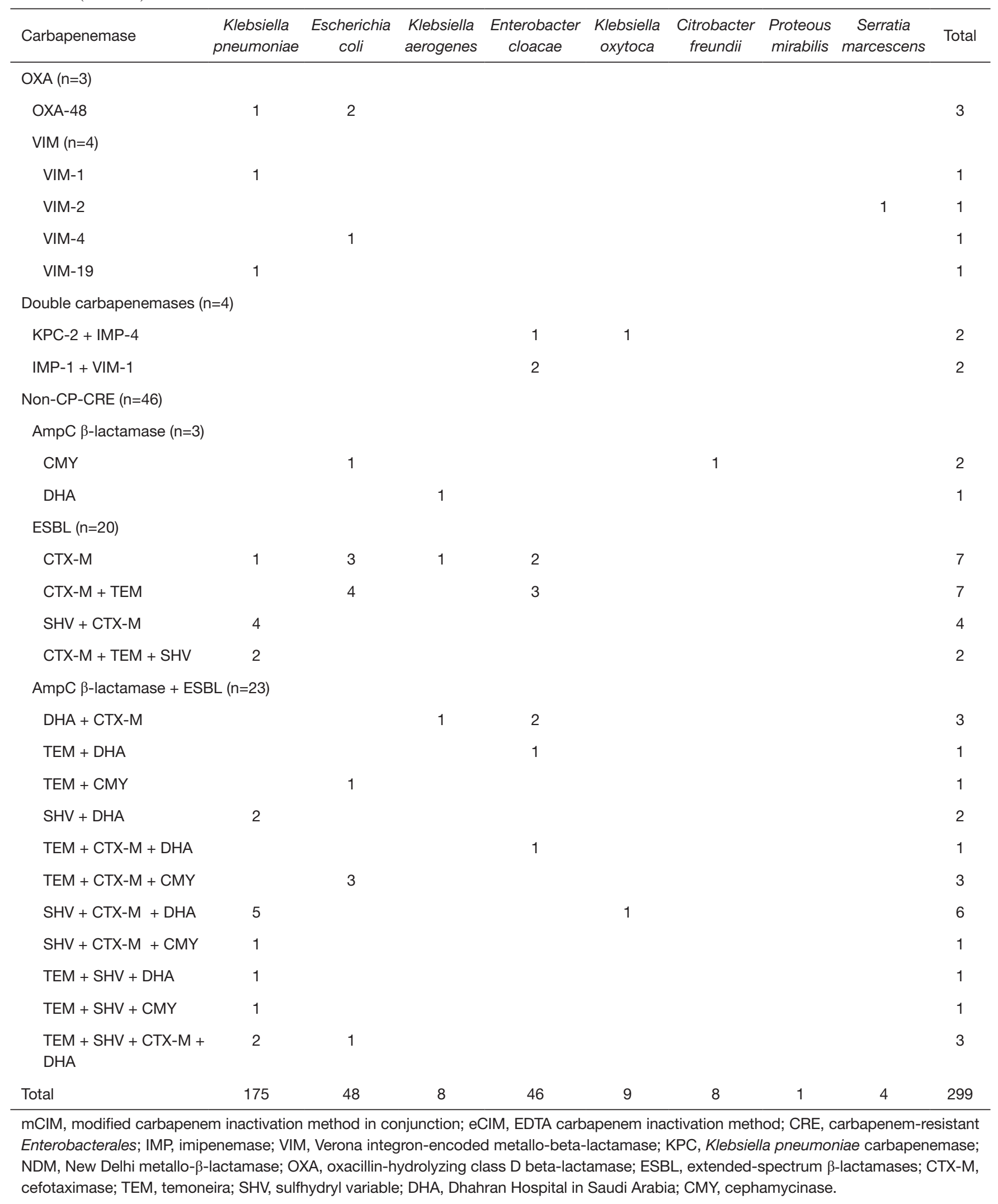


Table 2 Comparison of the performance of NG-Test CARBA 5 versus mCIM/eCIM for carbapenemase detection using 299 clinical CRE isolates

\begin{tabular}{|c|c|c|c|c|c|c|}
\hline & \multicolumn{2}{|c|}{ NG-Test CARBA 5} & \multicolumn{2}{|c|}{$\mathrm{mClM}$} & \multicolumn{2}{|c|}{ eCIM } \\
\hline All isolates & $100(98.1-100)$ & $99.9(99.5-100)$ & $99.6(97.5-100)$ & $100(90.4-100)$ & $97.4(90.1-99.5)$ & $100(97.3-100)$ \\
\hline $\mathrm{KPC}$ & $100(97.3-100)$ & $100(96.3-100)$ & $100(97.3-100)$ & $100(90.4-100)$ & NA & $100(97.3-100)$ \\
\hline NDM & $100(85.4-100)$ & $99.6(97.6-100)$ & $100(85.4-100)$ & $100(90.4-100)$ & $100(85.4-100)$ & $100(97.3-100)$ \\
\hline OXA-48 & $100(30.1-100)$ & $100(98.4-100)$ & $100(31.0-100)$ & $100(90.4-100)$ & NA & $100(31.0-100)$ \\
\hline VIM & $100(39.6-100)$ & $100(98.4-100)$ & 75 (21.9-98.7) & $100(90.4-100)$ & $100(31.0-100)$ & $100(97.3-100)$ \\
\hline KPC+IMP & $100(19.8-100)$ & $100(98.4-100)$ & $100(19.8-100)$ & $100(90.4-100)$ & $0(0-80.2)$ & $100(97.3-100)$ \\
\hline VIM+IMP & $100(19.8-100)$ & $100(98.4-100)$ & $100(19.8-100)$ & $100(90.4-100)$ & $100(19.8-100)$ & $100(97.3-100)$ \\
\hline E. cloacae & $100(89.1-100)$ & $99.5(96.7-100)$ & $100(88.3-100)$ & $100(62.9-100)$ & 96.9 (82.0-99.8) & $100(46.3-100)$ \\
\hline K. oxytoca & $100(62.9-100)$ & $100(88.0-100)$ & $100(59.8-100)$ & $100(5.5-100)$ & 85.7 (42.0-99.2) & $100(54.6-100)$ \\
\hline K. aerogenes & $100(46.3-100)$ & $100(87.7-100)$ & $100(46.3-100)$ & $100(31.0-100)$ & $100(5.5-100)$ & $100(39.6-100)$ \\
\hline C. freundii & $100(56.1-100)$ & $100(87.0-100)$ & $100(56.1-100)$ & $100(5.5-100)$ & $100(56.1-100)$ & NA \\
\hline S. marcescens & $100(39.6-100)$ & NA & 75 (21.9-98.7) & NA & NA & $100(31.0-100)$ \\
\hline P. mirabilis & $100(5.5-100)$ & NA & $100(5.5-100)$ & NA & NA & $100(5.5-100)$ \\
\hline
\end{tabular}

The numbers in bracket indicate 95\% confidence interval. mCIM, modified carbapenem inactivation method in conjunction; eCIM, EDTA carbapenem inactivation method; CRE, carbapenem-resistant Enterobacterales; NA, not available.

\section{Performances of NG-Test CARBA 5 and eCIM/mCIM in comparison with genotypic test}

Both NG-Test CARBA 5 and mCIM/eCIM showed excellent results in the carbapenemase detection compared with genotypic test. The performance of the two tests is illustrated in Table 2 and discrepant results are listed in Table 3. When calculating analytical specificity and sensitivity, each NG-Test CARBA 5 assay was considered as five individual tests, meaning a total of $1,495(299 \times 5)$ tests were conducted. The overall specificity and sensitivity of NG-Test CARBA 5 were $99.9 \%$ and $100 \%$, respectively. NG-Test CARBA 5 detected all KPC-, NDM-, VIM- and OXA-48-producing isolates perfectly. However, an IMP4 producer gave an additional weak false-positive signal for NDM, which makes the specificity for NDM decrease to $99.6 \%$. mCIM/eCIM achieved high specificity of $100 \% / 100 \%$ and sensitivity of $99.6 \% / 97.4 \%$, with one $S$. marcescens isolate harboring VIM-2 undetected. Additionally, two isolates genetically characterized as carrying both $b l a_{\mathrm{KPC}}$ and $b l a_{\text {IMP }}$ yielded mCIM-positive (zone diameter $=6 \mathrm{~mm}$ ) but eCIM-negative results (zone diameter $=6 \mathrm{~mm}$ ), which failed to report the presence of MBL. It is expected as EDTA does not inhibit KPC. Five IMP producers, which possessed relatively low level of carbapenem resistance compared with other isolates, were correctly identified by NG-Test CARBA 5 and mCIM/eCIM (Table 4). As for non-CP-CRE isolates harboring either ESBLs or AmpC $\beta$-lactamase, both tests yielded negative results. When it came to the performances of the two assays on different isolates, $K$. pneumoniae, E. coli, $K$. aerogenes, $C$. freundii and P. mirabilis were all correctly identified. NG-Test CARBA 5 failed in an E.cloacae isolate and $m$ CIM/eCIM failed in $S$. marcescens, E. cloacae and K. oxytoca isolates (Table 3).

\section{Discussion}

Rapid and accurate detection of CP-CRE is of great necessity for both treatment decision making and implementation of infection control. The main features of NG-Test CARBA5, mCIM/eCIM and genotypic test are 
Table 3 Characteristics of isolates showing discrepant phenotypic results with genotypic results

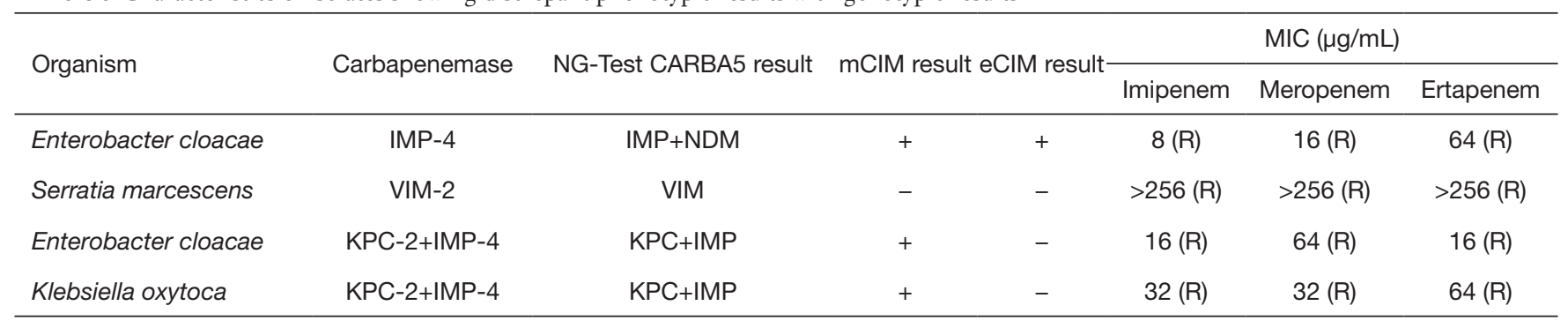

mCIM, modified carbapenem inactivation method in conjunction; eCIM, EDTA carbapenem inactivation method; IMP, imipenemase; VIM, Verona integron-encoded metallo-beta-lactamase; KPC, Klebsiella pneumoniae carbapenemase; NDM, New Delhi metallo- $\beta$-lactamase; R, resistant.

Table 4 Results of NG-Test CARBA 5 and mCIM/eCIM for the detection of CRE with relatively low level of carbapenem resistance

\begin{tabular}{|c|c|c|c|c|c|c|}
\hline Organism & Carbapenemase & $\begin{array}{c}\text { NG-Test CARBA } 5 \\
\text { result }\end{array}$ & $\begin{array}{l}\mathrm{mCIM} / \mathrm{eCIM} \\
\text { result }\end{array}$ & \multicolumn{3}{|c|}{$\mathrm{MIC}(\mu \mathrm{g} / \mathrm{mL})$} \\
\hline Klebsiella oxytoca & IMP-1 & + & + & $2(l)$ & $2(\mathrm{l})$ & $2(\mathrm{R})$ \\
\hline Klebsiella oxytoca & IMP-4 & + & + & $1(\mathrm{~S})$ & $1(\mathrm{~S})$ & $2(\mathrm{R})$ \\
\hline Klebsiella oxytoca & IMP-4 & + & + & $2(l)$ & $4(\mathrm{R})$ & $2(\mathrm{R})$ \\
\hline Klebsiella pneumoniae & IMP-4 & + & + & $2(I)$ & $4(\mathrm{R})$ & $2(\mathrm{R})$ \\
\hline
\end{tabular}

$\mathrm{mCIM}$, modified carbapenem inactivation method in conjunction; eCIM, EDTA carbapenem inactivation method; CRE, carbapenem-resistant Enterobacterales; IMP, imipenemase; MIC, minimum inhibitory concentration; I, intermediate; S, susceptible; R, resistant.

Table 5 Comparison of the main features of three carbapenemase detection methods

\begin{tabular}{|c|c|c|c|}
\hline Parameter & NG-Test CARBA 5 & $\mathrm{mCIM} / \mathrm{eCIM}$ & Xpert $^{\circledR}$ CARBA R \\
\hline Cost & Moderate & Low & High \\
\hline Special instruments required & No & No & Yes \\
\hline Experiment simplicity & Simple & Moderate & Simple \\
\hline Result interpretation & Simple & Moderate & Simple \\
\hline Cover range for carbapenemase & KPC, NDM, VIM, IMP, OXA-48-like & Serine carbapenemase, MBL & KPC, NDM, VIM, IMP, OXA-48-like \\
\hline
\end{tabular}

illustrated in Table 5. As every assay has its own limitations, two phenotypic assays were compared with genotypic test to choose suitable methods for the establishment of the CRE diagnostic pathways.

The CLSI guideline stated that mCIM demonstrated $>99 \%$ sensitivity and specificity, while eCIM showed $>95 \%$ sensitivity and $>92 \%$ specificity when detecting the five major types of carbapenemases among Enterobacterales isolates (20). Our results coincided with it, with sensitivity of mCIM and eCIM being $99.6 \%$ and $97.4 \%$, respectively, and specificity both being $100 \%$. mCIM/eCIM is able to identify rare carbapenemases like GES-4, IMP-6- and NDM-1, which are beyond the detection range of NGTest CARBA 5 (24). Additionally, result interpreting of 
$\mathrm{mCIM} / \mathrm{eCIM}$ is objective and convenient as it depends on a defined zone diameter. The majority of the isolates tested here exhibited a mCIM zone diameter of $6 \mathrm{~mm}$ and eCIM zone diameter of approximately $15 \mathrm{~mm}$, which confirmed its ease of interpretation. One limitation of $\mathrm{mCIM} / \mathrm{eCIM}$ is its inability to differentiate between MBLs and serine carbapenemases when the organism harbor both of them $(3,7)$. The number of organisms encoding double carbapenemases is enlarging, which is confirmed by an observation that the annual incidence of KPC-2/NDM-1 $K$. pneumoniae is increasing. The rate was zero from 2012 to 2015 and rose to $0.28 \%$ in 2016 , finally reaching $0.58 \%$ in 2017 (25). Due to the higher frequency of doubleenzyme producers, methods that can correctly identify between MBL and serine carbapenemases are of great need, as organism producing different enzymes leads to diverse therapies. Another limitation of mCIM/eCIM is it requires an overnight incubation which prolongs TATs.

NG-Test CARBA 5 is a newly developed assay for the carbapenemase detection. In this study, NG-Test CARBA 5 achieved high sensitivity of $100 \%$. Organisms co-producing serine cabapenemase and MBL were precisely differentiated, which is especially valuable as $\mathrm{mCIM} / \mathrm{eCIM}$ cannot identify them. The specificity of NG-Test CARBA 5 decreased to $99.9 \%$ due to an additional NDM false-positive band from an IMP-4 E. cloacae. False-positive results were rarely reported, with the exception of two wrong signals for VIM and KPC bands observed in another study evaluating 197 isolates (15). KPC and NDM are the two carbapenemases most frequently identified in Chinese hospitals (26-28). NG-Test CARBA 5 exhibited good performance with no false-negative but a false-positive result in their detection. NG-Test CARBA 5 showed advantages in the identification of OXA-48-like and IMP. The detection of OXA-48-like by phenotypic methods can be challenging as the hydrolysis activity of OXA-48-like is generally weak (29). NG-Test CARBA 5, based on the recognition of conserved epitopes of the carbapenemase, is not influenced by it and therefore has advantages in the detection for OXA-48-like. Except for OXA-48, NG-Test CARBA 5 has also correctly identified several OXA-48 variants, e.g., OXA-181, OXA-204, and OXA-162 (19). However, as it has been reported, the high sequence diversity within IMP family makes it more difficult to be detected (15). In this study, Organisms producing IMP-1, IMP-4, IMP-8, and IMP-26 were all correctly identified. With wide coverage for IMP variants, NG-Test CARBA 5 is a great tool for carbapenemase detection in areas where IMP is prevalent. One disadvantage for NG-
Test CARBA 5 is that despite being able to identify nearly all variants of NDM, KPC, VIM and OXA-48-like, rare carbapenemases are out of its detection range (17). For example, GIM, SME, IMI are undetectable by NG-Test CARBA $5(17,19)$.

CRE infection, especially CRE blood stream infection, is associated with high mortality. In a study reviewing bloodstream infection cases, patients with CRE bacteremia had a median of 52-hour from culture collection until receipt of active therapy and the 52-hour delay was associated with increased mortality. Additionally, 21\% of patients with CRE bacteremia did not receive active therapy as they died prior to the availability of antimicrobial susceptibility testing reporting (30). Therefore, rapid diagnosis of CRE is essential for early treatment of patients. As for the current CRE diagnostic pathway in PUMCH, antimicrobial susceptibility testing is performed firstly after the bacterial identification, and then mCIM/eCIM is conducted to CRE isolate for the confirmation of its carbapenem-resistant mechanisms. It takes approximately $48 \mathrm{~h}$ from sample reception to the reporting of antimicrobial susceptibility testing and results for carbapenemase testing require an additional night.

A major limitation of this study is that cabapenemases that could not be detected by NG-Test CARBA 5 were not fully covered. In addition, this study focused only on Enterobacterales. However, non-fermenters like Acinetobacter baumannii and Pseudomonas aeruginosa are also frequently encountered organisms which produce carbapenemase in the clinical practice. The performance of NG-Test CARBA 5 and mCIM/eCIM on these organisms should be further investigated. What's more, the diversity of carbapenemases tested in this study is limited. Only 4 VIM-producing isolates and 3 OXA-producing isolates were included, while 172/253 were KPC producers. More VIM and OXA isolates should be tested in further study.

\section{Acknowledgments}

Funding: This study was supported by National Natural Science Foundation of China (82072318), National Key Research and Development Program of China (2018YFE0101800, 2018YFC1200105), Beijing Key Clinical Specialty for Laboratory Medicine - Excellent Project (No. ZK201000). The funders were not involved in the study design, collection, analysis, interpretation of data, writing of this article, or the decision to submit it for publication. 


\section{Footnote}

Reporting Checklist: The authors have completed the STARD reporting checklist. Available at http://dx.doi.org/10.21037/ atm-20-8216

Data Sharing Statement: Available at http://dx.doi. org/10.21037/atm-20-8216

Conflicts of Interest: All authors have completed the ICMJE uniform disclosure form (available at http://dx.doi. org/10.21037/atm-20-8216). The authors have no conflicts of interest to declare.

Ethical Statement: The authors are accountable for all aspects of the work in ensuring that questions related to the accuracy or integrity of any part of the work are appropriately investigated and resolved. The study was conducted in accordance with the Declaration of Helsinki (as revised in 2013). The study was approved by The Human Research Ethics Committee of Peking Union Medical College Hospital of S-K1056 and informed consent was waived as the study collected remaining samples from clinical tested samples without extra operation of patients.

Open Access Statement: This is an Open Access article distributed in accordance with the Creative Commons Attribution-NonCommercial-NoDerivs 4.0 International License (CC BY-NC-ND 4.0), which permits the noncommercial replication and distribution of the article with the strict proviso that no changes or edits are made and the original work is properly cited (including links to both the formal publication through the relevant DOI and the license). See: https://creativecommons.org/licenses/by-nc-nd/4.0/.

\section{References}

1. Shaikh N, Drego L, Shetty A, et al. Comparative evaluation of Xpert(r) Carba-R assay with conventional methods for detection of carbapenemase producing enterobacteriaceae. International Journal of Infectious Diseases 2016;45:156.

2. Tamma PD, Simner PJ. Phenotypic Detection of Carbapenemase-Producing Organisms from Clinical Isolates. J Clin Microbiol 2018;56:e01140-18.

3. Jenkins S, Ledeboer NA, Westblade LF, et al. Evaluation of NG-Test Carba 5 for Rapid Phenotypic Detection and
Differentiation of Five Common Carbapenemase Families: Results of a Multicenter Clinical Evaluation. J Clin Microbiol 2020;58:e00344-20.

4. Tamma PD, Opene BN, Gluck A, et al. Comparison of 11 Phenotypic Assays for Accurate Detection of Carbapenemase-Producing Enterobacteriaceae. J Clin Microbiol 2017;55:1046-55.

5. Findlay J, Hopkins KL, Meunier D, et al. Evaluation of three commercial assays for rapid detection of genes encoding clinically relevant carbapenemases in cultured bacteria. J Antimicrob Chemother 2015;70:1338-42.

6. Zhanel GG, Lawson CD, Adam H, et al. Ceftazidimeavibactam: a novel cephalosporin/ $\beta$-lactamase inhibitor combination. Drugs 2013;73:159-77.

7. Sfeir MM, Hayden JA, Fauntleroy KA, et al. EDTAModified Carbapenem Inactivation Method: a Phenotypic Method for Detecting Metallo- $\beta$-Lactamase-Producing Enterobacteriaceae. J Clin Microbiol 2019;57:e01757-18.

8. Antonelli A, Giani T, Di Pilato V, et al. KPC-31 expressed in a ceftazidime/avibactam-resistant Klebsiella pneumoniae is associated with relevant detection issues. J Antimicrob Chemother 2019;74:2464-6.

9. Novelli A, Del Giacomo P, Rossolini GM, et al. Meropenem/vaborbactam: a next generation $\beta$-lactam $\beta$-lactamase inhibitor combination. Expert Rev Anti Infect Ther 2020;18:643-55.

10. Bonomo RA, Burd EM, Conly J, et al. CarbapenemaseProducing Organisms: A Global Scourge. Clin Infect Dis 2018;66:1290-7.

11. Pierce VM, Simner PJ, Lonsway DR, et al. Modified Carbapenem Inactivation Method for Phenotypic Detection of Carbapenemase Production among Enterobacteriaceae. J Clin Microbiol 2017;55:2321-33.

12. Zhou M, Wang D, Kudinha T, et al. Comparative Evaluation of Four Phenotypic Methods for Detection of Class A and B Carbapenemase-Producing Enterobacteriaceae in China. J Clin Microbiol 2018;56:e00395-18.

13. Baeza LL, Pfennigwerth N, Greissl C, et al. Comparison of five methods for detection of carbapenemases in Enterobacterales with proposal of a new algorithm. Clin Microbiol Infect 2019;25:1286.e9-1286.e15.

14. Kieffer N, Poirel L, Nordmann P. Rapid immunochromatography-based detection of carbapenemase producers. Infection 2019;47:673-5.

15. Hopkins KL, Meunier D, Naas T, Volland H, Woodford N. Evaluation of the NG-Test CARBA 5 multiplex immunochromatographic assay for the detection of KPC, 
OXA-48-like, NDM, VIM and IMP carbapenemases. J

Antimicrob Chemother 2018;73:3523-6.

16. Potron A, Fournier D, Emeraud C, et al. Evaluation of the Immunochromatographic NG-Test Carba 5 for Rapid Identification of Carbapenemase in Nonfermenters. Antimicrob Agents Chemother 2019;63:e00968-19.

17. Bodendoerfer E, Keller PM, Mancini S. Rapid identification of NDM-, KPC-, IMP-, VIM- and OXA48-like carbapenemase-producing Enterobacteriales from blood cultures by a multiplex lateral flow immunoassay. J Antimicrob Chemother 2019;74:1749-51.

18. Giordano L, Fiori B, D'Inzeo T, et al. Simplified Testing Method for Direct Detection of CarbapenemaseProducing Organisms from Positive Blood Cultures Using the NG-Test Carba 5 Assay. Antimicrob Agents Chemother 2019;63:e0550-19.

19. Takissian J, Bonnin RA, Naas T, et al. NG-Test Carba 5 for Rapid Detection of Carbapenemase-Producing Enterobacterales from Positive Blood Cultures. Antimicrob Agents Chemother 2019;63:e00011-19.

20. CLSI. Performance Standards for Antimicrobial Susceptibility Testing. 30th ed. CLSI supplement M100. Wayne, PA: Clinical and Laboratory Standards Institute; 2020.

21. Poirel L, Walsh TR, Cuvillier V, Nordmann P. Multiplex PCR for detection of acquired carbapenemase genes. Diagn Microbiol Infect Dis 2011;70:119-23.

22. Founou LL, Founou RC, Allam M, et al. Genome Sequencing of Extended-Spectrum $\beta$-Lactamase (ESBL)Producing Klebsiella pneumoniae Isolated from Pigs and Abattoir Workers in Cameroon. Front Microbiol 2018;9:188.

23. Tewari R, Mitra S, Ganaie F, et al. Dissemination and

Cite this article as: Zhu Y, Jia P, Li X, Wang T, Zhang J, Zhang G, Duan S, Kang W, Xu Y, Yang Q. Carbapenemase detection by NG-Test CARBA 5-a rapid immunochromatographic assay in carbapenem-resistant Enterobacterales diagnosis. Ann Transl Med 2021;9(9):769. doi: 10.21037/atm-20-8216 characterisation of Escherichia coli producing extendedspectrum $\beta$-lactamases, AmpC $\beta$-lactamases and metallo- $\beta$ lactamases from livestock and poultry in Northeast India: A molecular surveillance approach. J Glob Antimicrob Resist 2019;17:209-15.

24. Kuchibiro T, Komatsu M, Yamasaki K, et al. Evaluation of the modified carbapenem inactivation method for the detection of carbapenemase-producing Enterobacteriaceae. J Infect Chemother 2018;24:262-6.

25. Gao H, Liu Y, Wang R, et al. The transferability and evolution of NDM-1 and KPC-2 co-producing Klebsiella pneumoniae from clinical settings. EBioMedicine 2020;51:102599.

26. Miao M, Wen H, Xu P, et al. Genetic Diversity of Carbapenem-Resistant Enterobacteriaceae (CRE) Clinical Isolates From a Tertiary Hospital in Eastern China. Front Microbiol 2019;9:3341.

27. Zhou H, Zhang K, Chen W, et al. Epidemiological characteristics of carbapenem-resistant Enterobacteriaceae collected from 17 hospitals in Nanjing district of China. Antimicrob Resist Infect Control 2020;9:15.

28. Zhang R, Liu L, Zhou H, et al. Nationwide Surveillance of Clinical Carbapenem-resistant Enterobacteriaceae (CRE) Strains in China. EBioMedicine 2017;19:98-106.

29. Strich JR, Wang H, Cissé OH, et al. Identification of the OXA-48 Carbapenemase Family by Use of Tryptic Peptides and Liquid Chromatography-Tandem Mass Spectrometry. J Clin Microbiol 2019;57:e01240-18.

30. Satlin MJ, Cohen N, Ma KC, et al. Bacteremia due to carbapenem-resistant Enterobacteriaceae in neutropenic patients with hematologic malignancies. J Infect 2016;73:336-45. 